Please do not remove this page

RMIT

UNIVERSITY

\title{
Systematic design approach for optimized resonantly enhanced Mach-zehnder modulators
}

Visagathilagar, Yuvaraja; Nguyen, Giang; Mitchell, Arnan; Austin, Michael

https://researchrepository.rmit.edu.au/esploro/outputs/9921862018201341/filesAndLinks?institution=61RMIT_INST\&index=null

Visagathilagar, Y., Nguyen, G., Mitchell, A., \& Austin, M. (2006). Systematic design approach for optimized resonantly enhanced Mach-zehnder modulators. IEEE Journal of Lightwave Technology, 24(1), 555-562. https://doi.org/10.1109/jlt.2005.860162

Published Version: https://doi.org/10.1109/jlt.2005.860162

Repository homepage: https://researchrepository.rmit.edu.au

(c) 2006 IEEE. Personal use of this material is permitted. However, permission to reprint/republish this material for advertising or promotional purposes or for creating new collective works for resale or redistribution to servers or lists, or to reuse any copyrighted component of this work in other works must be obtained from the IEEE.

Downloaded On 2023/04/26 09:49:38 +1000 


\title{
Systematic Design Approach for Optimized Resonantly Enhanced Mach-Zehnder Modulators
}

\author{
Yuvaraja S. Visagathilagar, Member, IEEE, Thach G. Nguyen, Arnan A. Mitchell, Member, IEEE, and
} Michael W. Austin, Member, IEEE

\begin{abstract}
A systematic design approach using the developed numerical model for the investigation of any arbitrary electrooptic modulator configuration is described, and its application to the simulation and synthesis of resonantly enhanced Mach-Zehnder modulators (RE-MZMs) is demonstrated. The tool is implemented using equivalent circuit model using transmission lines, lumped elements, and $N$-port $S$-parameters. The numerical tool is used to simulate the modulation enhancement factor and radio frequency (RF) return loss of a number of theoretically and experimentally demonstrated examples. Finally, the design tool is used to synthesize a new optimized RE-MZM. This RE-MZM is fabricated and measured, and predicted results are compared.
\end{abstract}

Index Terms-Design approach, $\mathrm{LiNbO}_{3}$, narrow bandwidth, numerical optimization, optical modulator, resonant enhancement.

\section{INTRODUCTION}

$\mathbf{I}$ $\mathrm{N}$ MANY telecommunications and military applications, such as in wideband fiber-optic signal distribution [1]-[3], $\mathrm{LiNbO}_{3}$ modulators are essential. For defense applications, broad bandwidth is essential. $\mathrm{LiNbO}_{3}$ modulators have been demonstrated in defense environment with exceptional bandwidth but only modest modulation efficiency. In many telecommunication applications, such as radio on fiber [4], only a narrow bandwidth is required with optimal efficiency being a priority.

Several narrow bandwidth optical modulators have been proposed in the literature to provide improved modulation efficiency at the expense of bandwidth. This are called resonantly enhanced Mach-Zehnder modulators (RE-MZMs) [5]-[9]. A few examples of RE-MZMs where optical resonators are used have been reported [10]. This investigation will be restricted to those modulators that use resonant radio frequency (RF) electrodes and no optical resonators.

Resonant electrodes can have complex forms with multiple resonant sections and interactions. Simulation of these electrodes, thus, requires rigorous transmission network analysis and subsequent modeling of the electrooptic interaction in the active region.

Currently, the tools for numerical modeling and design of microwave photonic devices, in particular, RE-MZMs are lim-

Manuscript received June 14, 2005; revised August 28, 2005. This work was supported by the Research Investment Fund (RIF) Grant, RMIT University.

The authors are with the Microelectronics and Materials Technology Centre (MMTC), School of Electrical and Computer Engineering, Royal Melbourne Institute of Technology (RMIT), Melbourne, Vic. 3001, Australia (e-mail: yuvaraja@rmit.edu.au; s9510300@student.rmit.edu.au; arnan. mitchell@rmit.edu.au; mwa@ @rmit.edu.au).

Digital Object Identifier 10.1109/JLT.2005.860162 ited. Many sophisticated numerical tools based on transmission line or network models can evaluate RF performance of electronic circuits with arbitrary configuration. Some of the commercially available tools includes HP-EEsof, Agilent ADS, Ansoft Microwave Office, SPICE, and Ansoft Serenade. Similar commercial simulation tools exist for the design of photonic systems and devices namely VPIsystems Transmission Maker and Optiwave BPM-CAD.

In the literature, numerical tools to model RE-MZMs have been investigated [6], [8], [11]-[13], however, they are limited to the approximations of the network models and also derived as approximated closed-form solutions. These tools calculated the electrooptic performance of the RE-MZMs, however, these models provide insufficient accuracy due to the complexity of the electrode structures.

Flexible numerical tools [14], [15], which can simulate both complex RF circuit behavior and electrooptic interaction, have been demonstrated. These models are based on a network configuration to accurately predict frequency-dependent electrooptic interactions using only network models of microwave/RF components. The models calculate the accumulated induced phase shift over the electrode cavity in relation to the optical path.

In this paper, the tool from [14] is extended to include general $N$-port S-parameter electrical networks. This tool is then used to analyze a range of previously published resonantly enhanced modulator structures. Finally, the numerical tool is used as a design approach, with the aid of numerical optimization, to synthesize a resonantly enhanced modulator. This modulator is realized and demonstrated. The measured performance is compared with the predicted performance in order to verify the accuracy and flexibility of the presented numerical tool and design approach.

This paper is structured as follows. In Section II, the theory of the numerical model used as a design approach is presented. In Section III, the developed numerical model is validated, with examples of RE-MZMs published in the literature for modulation enhancement factor and RF return loss. In Section IV, an optimized RE-MZM device is designed using this numerical model. The proposed numerical model is validated with an experimental implementation of this optimized RE-MZM device. Finally, a summary of the investigation is presented in Section V.

\section{Theory of the Numerical Network Model}

A conventional high-speed optical modulator is shown in Fig. 1(a). The RF electrode of the modulator is a traveling-wave 
(a)

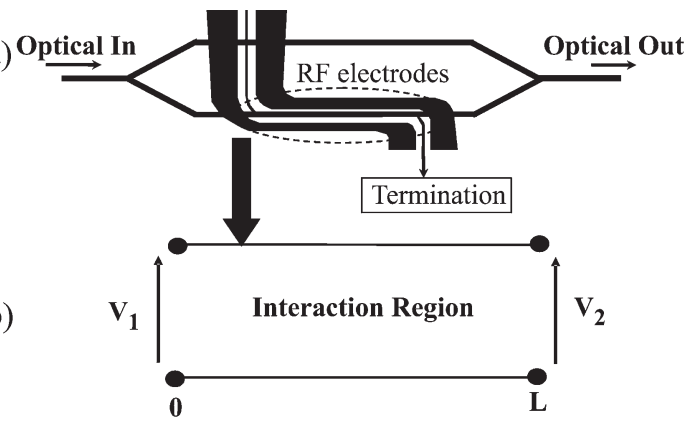

Fig. 1. Concept model of an optical modulator. (a) Device configuration. (b) Equivalent transmission line model.

electrode of the form using a coplanar-waveguide (CPW) structure. The electrode has an active section that interacts with the optical field and inactive sections that include the tapers and bends required for connectorizing to an external package.

These CPW sections can be approximated using a transmission line model with characteristics such as impedance $\left(Z_{o}\right)$, attenuation $(\alpha)$ per unit length, and effective microwave index $\left(N_{m}\right)$ [16], [17]. The equivalent model of the modulator is shown in Fig. 1(b). The complex amplitude of the voltage and current at each circuit node can be modeled using available RF network models; however, to model the electrooptic interaction requires further calculation.

To calculate the electrooptic interaction, it is necessary to calculate the accumulated phase shift induced through the application of an electric field along the length of the interaction region. The accumulated phase shift can be calculated by integrating the complex voltage along the interaction length, in the frame of reference of the optical carrier. When the active region is a length of uniform transmission line as shown in Fig. 1(b), the induced phase shift can be written as

$$
\Delta \phi=\frac{\pi}{V_{\pi} L} \int_{0}^{L}\left(A e^{+j \gamma z}+B e^{-j \gamma z}\right) e^{+j k_{o} N_{o} z} d z
$$

where

$$
\begin{aligned}
& A=\frac{V_{2}-V_{1} e^{-j \gamma L}}{2 j \sin (\gamma L)} \\
& B=V_{1}-A
\end{aligned}
$$

and

$$
\gamma=k_{m} N_{m}-j\left(\alpha_{o} \sqrt{f_{m}}+\alpha_{d} f_{m}\right) .
$$

The transmission line length is $L, V_{1}$ and $V_{2}$ are the complex voltages at $z=0$ and $z=L$, respectively, $V_{\pi}$ is the switch ON-OFF voltage of the modulator, $N_{m}$ and $N_{o}$ are the effective indices of the RF and optical waves, $k_{m}$ and $k_{o}$ are the wave numbers of the RF and optical waves $\left(k_{m}=2 \pi f_{m} / c\right)$, where $f_{m}$ is the modulating RF frequency, $\alpha_{o}$ and $\alpha_{d}$ are the coefficient of conductor loss and RF dielectric loss in $\mathrm{LiNbO}_{3}$, respectively. The optical effective index $N_{o}$ is computed using scalar finite difference tool based on the titaniumdiffused waveguide structure on $\mathrm{LiNbO}_{3}$ and corresponds to

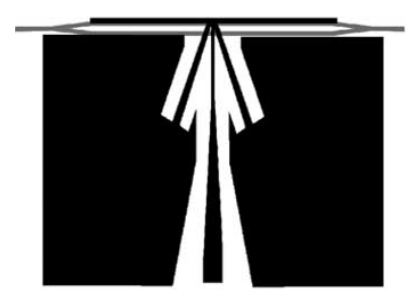

(a)

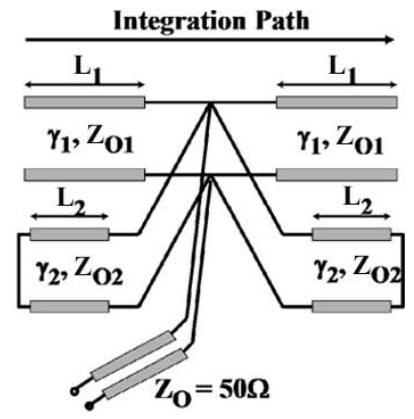

(b)
Fig. 2. Schematic of an RE-MZM with a double-stub electrode structure [7]. (a) Top view of the device. (b) Equivalent circuit model.

approximately 2.15 at optical wavelength of $1550 \mathrm{~nm}$. Thus, the modulation is proportional to the induced phase difference between the arms of the MZM.

To illustrate the simulation process, an example of an REMZM [7] published in the literature is shown in Fig. 2(a). An equivalent transmission line model is shown in Fig. 2(b), which represents the electrode structure. The complex potential can be calculated using available RF network models, and this potential can be used to calculate the induced phase shift given by (1).

The resonant modulator is represented as a network of active and inactive sections. The complex potentials calculated at the nodes of the network model in Fig. 2(b) are used to calculate the potentials along the length of each section. The attenuation, impedance, and effective index calculated using a finite element model (FEM) [18] enable the calculation of the complex potential at each node along each section of the electrode. An electrostatic FEM can be used to calculate the relationship between the complex potential and the field strength across the active region cross section.

An overlap between the guided optical mode calculated using a finite difference modeling (FDM) [19] and the static electric field in the active region, along with the electrooptic coefficient, can be used to calculate the relationship between optical phase shift and applied electric potential. The placement of the RF electrode on the optical waveguides is essential to find the optimum efficiency represented by $V_{\pi}$ as reported in [20] and given by (1). Thus, the computed induced phase shift is a function of the optimum placement of the RF electrode, the electrode characteristics, and the optical waveguide characteristics. For a given active region, electrode and optical waveguide configuration, the FEM modeling can be conducted ahead of time.

To calculate the modulation characteristics, it is necessary to calculate the accumulated phase shift along the length of the active region. This can be done by integrating the instantaneous phase shift calculated at each point along the active region. The integration path used for the example of Fig. 2 is shown in Fig. 2(b). When biased at quadrature, the gain [or modulation enhancement factor (MEF)] of the modulator can be calculated as

$$
\mathrm{MEF}=20 \log _{10}\left(\frac{\Delta \phi_{r}}{\Delta \phi_{u}}\right)
$$




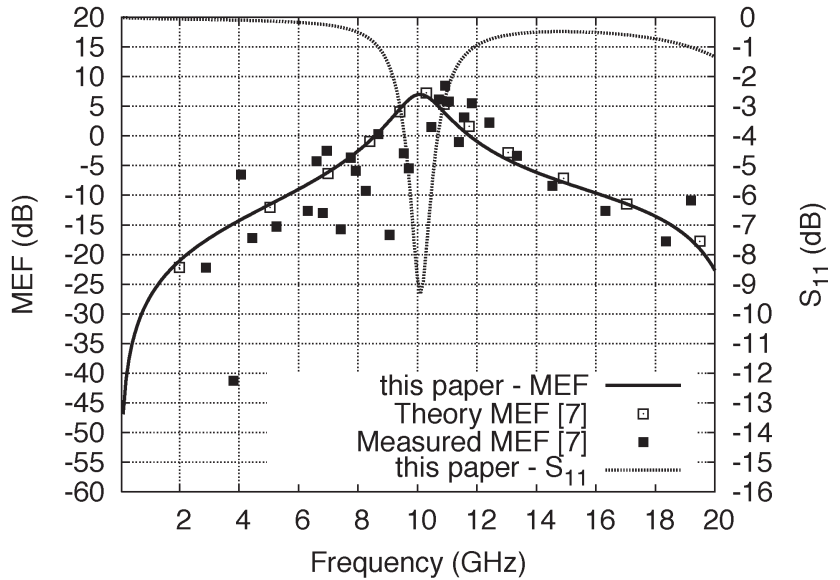

Fig. 3. Simulated MEF and $S_{11}$ including the reported results of [7] of the structure shown in Fig. 2.

where the induced phase shift of a resonant modulator and similar unenhanced modulator terminated with a $50-\Omega$ source is represented by $\left(\Delta \phi_{r}\right)$ and $\left(\Delta \phi_{u}\right)$, respectively, and, thus, these phase shifts and the overlap are related to the $V_{\pi}$ of a particular electrode placement on the optical modulator given by (1).

Our model allows simulation of electrodes formed by transmission line networks of arbitrary complexity, with arbitrary configuration of active regions through definition of possible multiple integral paths among these transmission lines. In this way, the bulk of all reported resonant modulators can be accurately simulated.

\section{VAlidation OF NumericAl Model AgAinst Published StRuCTURES}

To validate the accuracy and flexibility of the presented numerical model as a design approach, the behavior of various reported RE-MZMs [6]-[9] will be analyzed in the following sections. The tool will be used to simulate the MEF and RF return loss performance of each RE-MZM structure. The performance predicted using the tool presented in Section II is compared to the measured and simulated performance in literature.

\section{A. Double-Stub Resonant Modulator}

In Fig. 2(a), the RE-MZM has a resonant cavity consisting of two open-ended resonators and impedance matched using shorted double-stub structures in its central section [7]. The equivalent model of the structure is shown in Fig. 2(b).

The reported transmission line characteristic of the equivalent model depicted in Fig. 2(b) were $Z_{o 1}=70.1 \Omega, Z_{o 2}=$ $28.7 \Omega, L_{1}=0.1624 \mathrm{~mm}, L_{2}=0.8732 \mathrm{~mm}, N_{m 1}=3.51$, $N_{m 2}=4.13, \alpha_{1}=0.71 \mathrm{~dB} /(\mathrm{cm} \sqrt{\mathrm{GHz}})$, and $\alpha_{2}=0.39 \mathrm{~dB} /$ $(\mathrm{cm} \sqrt{\mathrm{GHz}})$, respectively. The input of the structure is connected to a $50-\Omega$ source.

The induced phase shift of the RE-MZM device was calculated using the numerical model in Section II. The MEF of the device was calculated using (2). The simulated MEF and RF return loss $\left(\left|S_{11}\right|\right)$ as a function of frequency is shown in Fig. 3.

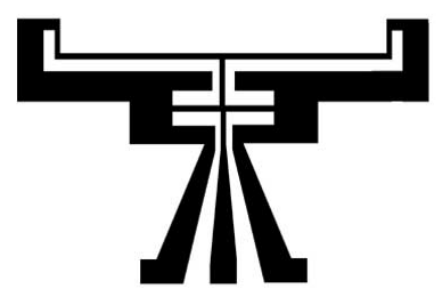

(a)

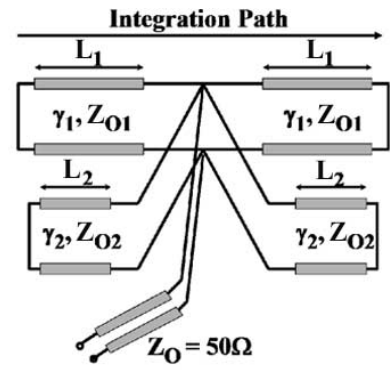

(b)
Fig. 4. RE-MZM 2-short-path resonant structure with shorted-end resonators [8]. (a) Top view of the device. (b) Equivalent circuit model.

For comparison, the reported theory and measured MEF has been included in Fig. 3.

The model in Section II predicts an MEF of $7 \mathrm{~dB}$ operating at $10 \mathrm{GHz}$ as shown in Fig. 3. The investigation in [7] predicted an MEF of $8.8 \mathrm{~dB}$, while the demonstrated performance was $7.7 \mathrm{~dB}$ at $10 \mathrm{GHz}$. The predicted MEF in Section II and the predicted MEF in the literature provide similar performance. The predicted induced phase shift presented in [7] was derived in an approximate closed form. Thus, the model proposed in Section II provides a more realistic and flexible model to represent an RE-MZM structure as a network model. The proposed numerical model predicts that the device structure has an $S_{11}$ of approximately $-10 \mathrm{~dB}$. However, [7] does not provide a predicted or measured RF return loss.

\section{B. Short-Path Resonant Modulator}

Another example of an RE-MZM is shown in Fig. 4, where short-ended resonators form the cavities [8], and similar to the example in Section III-A, a shorted double-stub structure provides impedance matching. The integration path of the device is between the shorted transmission line sections.

In this example, the reported transmission line characteristics of the equivalent model as depicted in Fig. 4(b) were $Z_{o 1}=35 \Omega, Z_{o 2}=35 \Omega, L_{1}=0.8 \mathrm{~mm}, L_{2}=1.12 \mathrm{~mm}$, $N_{m 1}=2.7, N_{m 2}=2.7, \alpha_{1}=0.35 \mathrm{~dB} /(\mathrm{cm} \sqrt{\mathrm{GHz}})$, and $\alpha_{2}=$ $0.35 \mathrm{~dB} /(\mathrm{cm} \sqrt{\mathrm{GHz}})$, respectively. The input of the structure is connected to a $50-\Omega$ source. The MEF of the device was calculated using (2). The simulated MEF and RF return loss $\left(\left|S_{11}\right|\right)$ using the numerical model in Section II is shown in Fig. 5. For comparison, the reported theory and measured MEF and $S_{11}$ have been included in Fig. 5.

The predicted MEF using the model given in Section II is approximately $7.2 \mathrm{~dB}$ at $25 \mathrm{GHz}$. In [8], the predicted and measured MEF is $\sim 10$ and $6 \mathrm{~dB}$, respectively, at $25 \mathrm{GHz}$. The large discrepancy in the predicted MEF in [8] and the predicted MEF in Section II can be attributed to the approximate closedform model used in [8] for the analysis of the induced phase shift. However, the simulation using the model in Section II and depicted in Fig. 5 provides an excellent match to the measured result in [8], since the model in Section II was a more realistic model considering all aspects of the device structure.

The RF return loss of this example using the model in Section II is $-4.5 \mathrm{~dB}$. The predicted and measured $S_{11}$ of [8] 


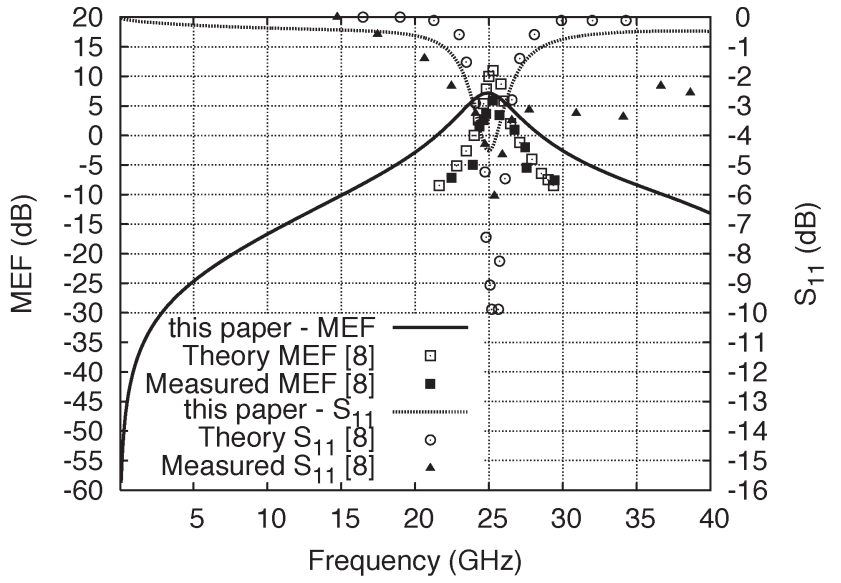

Fig. 5. Simulated MEF and $S_{11}$ including the reported results of [8] of the structure shown in Fig. 4.

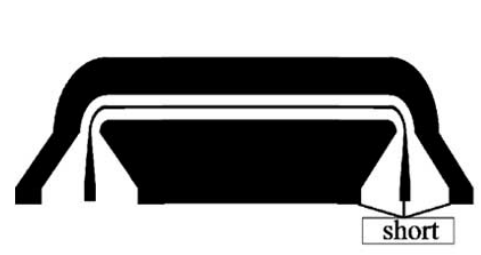

(a)

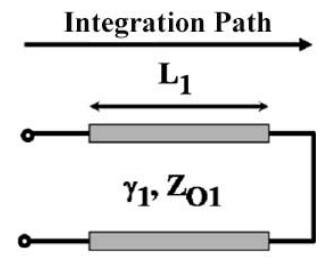

(b)
Fig. 6. RE-MZM 3-single-end shorted resonant structure [6]. (a) Top view of the device. (b) Equivalent circuit model.

are approximately -10 and $-6 \mathrm{~dB}$, respectively. It can be seen that the predicted numerical result in Section II as depicted in Fig. 5 has a similar value to the measured $S_{11}$. This result complements the result observed on the MEF above.

\section{Single-End Shorted Resonant Modulator}

In this example, a conventional broadband optical modulator is applied with a short circuit at the output port of the electrode [6], thus, forming a single-pass resonant modulator as shown in Fig. 6. The input port of the modulator is connected to a $50-\Omega$ source, thus, this does not provide a strong cavity, since the input port has only a small mismatch.

The reported transmission line characteristics of the equivalent model as depicted in Fig. 6(b) were $Z_{o 1}=35 \Omega$, $L_{1}=24 \mathrm{~mm}, N_{m 1}=2.21$, and $\alpha_{1}=0.6 \mathrm{~dB} /(\mathrm{cm} \sqrt{\mathrm{GHz}})$, respectively. The MEF of the device was calculated using (2). The simulated MEF and RF return loss $\left(\left|S_{11}\right|\right)$ using the model in Section II are shown in Fig. 7. The reported theory and measured MEF have been included in Fig. 7 for comparison.

The predicted MEF of this example using the model in Section II is approximately $5.2 \mathrm{~dB}$ at about $1 \mathrm{GHz}$. The predicted and measured MEF in [6] are 3.5 and $4.5 \mathrm{~dB}$, respectively, at $1 \mathrm{GHz}$. The predicted result in Section II and the reported measurement show good agreement, thus, validating the accuracy of the proposed model. However, as reported [6], the predicted value is lower, since the model was an approximate closed-form tool for the induced phase shift. It can be observed that the simulated MEF is negligible over $5 \mathrm{GHz}$, since the attenuation in the cavity is large and, thus,

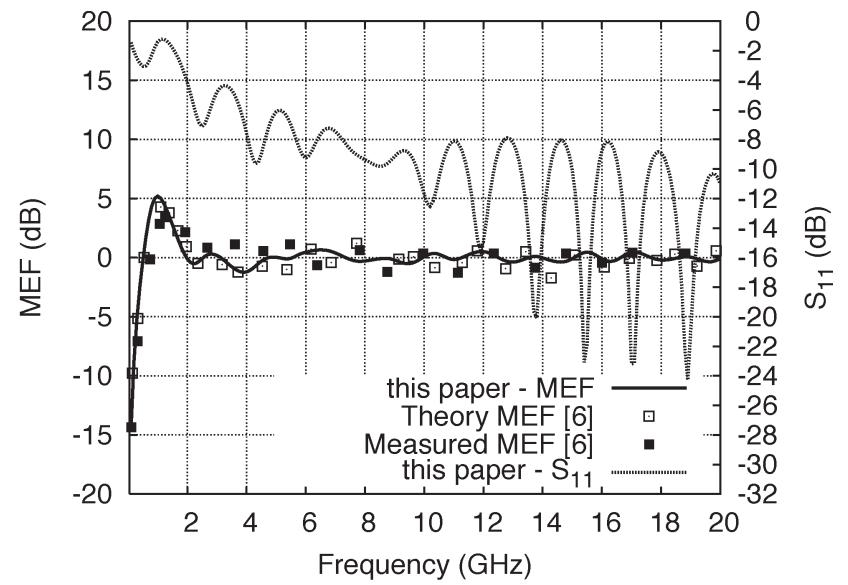

Fig. 7. Simulated MEF and $S_{11}$ including the reported results of [6] of the structure shown in Fig. 6.

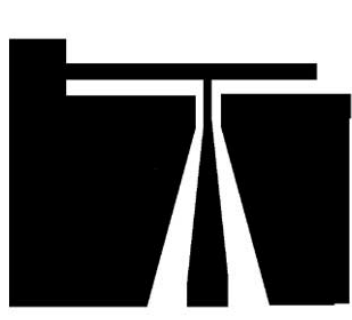

(a)

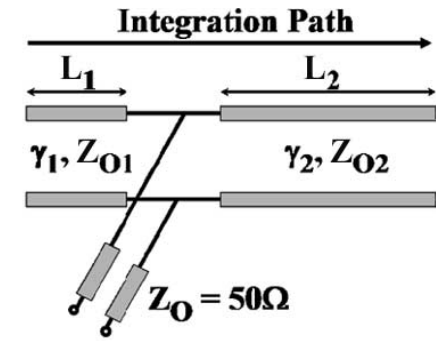

(b)
Fig. 8. RE-MZM 4-asymmetric resonant structure with mixed open and short-end resonators [9]. (a) Top view of the device. (b) Equivalent circuit model.

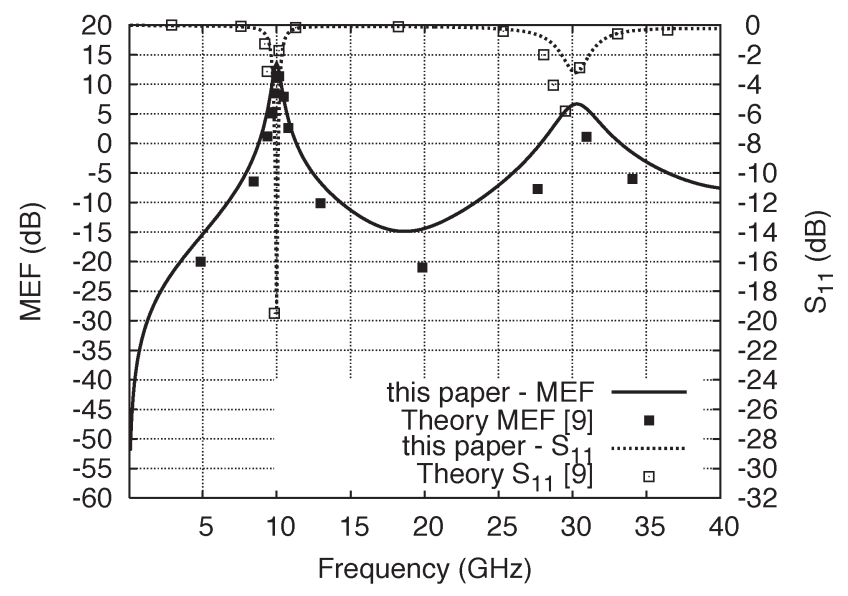

Fig. 9. Simulated MEF and $S_{11}$ including the reported results of [9] of the structure shown in Fig. 8.

the modulation efficiency is similar to the broadband optical modulator terminated with a $50-\Omega$ load.

The $S_{11}$ of the RE-MZM using the model in Section II is $-1.2 \mathrm{~dB}$ at $1 \mathrm{GHz}$. The literature does not report the measurement and predicted value for the RF return loss. However, it can be explained that the device will not have a good $S_{11}$, since the input is matched, and, thus, all the reflected power from the output port will be seen at the input port. Therefore, the predicted $S_{11}$ will be small as shown in Fig. 7 . 
TABLE I

Summary of the Simulated And Reported MEF And $S_{11}$ For the VARIous RE-MZMs Presented

\begin{tabular}{lcccccc}
\hline \multicolumn{1}{c}{ Configurations } & $\begin{array}{c}\text { Numerical - this work } \\
\text { (predicted) } \\
\text { (dB) }\end{array}$ & \multicolumn{2}{c}{$\begin{array}{c}\text { Reported } \\
\text { (measured) }\end{array}$} & \multicolumn{2}{c}{$\begin{array}{c}\text { Reported } \\
\text { (predicted) } \\
\text { (dB) }\end{array}$} & \multicolumn{2}{c}{$\begin{array}{c}\text { (dB) } \\
\end{array}$} & $M E F$ & $S_{11}$ & $M E F$ & $S_{11}$ & $M E F$ & $S_{11}$ \\
\hline \hline RE-MZM 1 [7] at 10 GHz & 7 & -10 & 8.8 & - & 7.7 & - \\
RE-MZM 2 [8] at 25 GHz & 7.2 & -4.5 & 6 & -6 & $>10$ & -10 \\
RE-MZM 2 [6] at 1 GHz & 5.2 & -1.2 & 4.5 & - & 3.5 & - \\
RE-MZM 2 [9] at $10 \mathrm{GHz}$ & 13.5 & -19 & - & - & 11.4 & -20 \\
\hline
\end{tabular}

\section{Asymmetric Resonant Modulator}

As a final example, an RE-MZM is composed of mixed resonators using open and short-end terminations to form a resonant cavity [9] as shown in Fig. 8. The impedance matching is achieved by the placement of the input port along the resonator.

In this example, the reported transmission line characteristics of the equivalent model as depicted in Fig. 8(b) were $Z_{o 1}=$ $34.71 \Omega, Z_{o 2}=34.71 \Omega, L_{1}=0.21 \mathrm{~mm}, L_{2}=1.55 \mathrm{~mm}$, $N_{m 1}=4.258, N_{m 2}=4.258, \alpha_{1}=0.309 \mathrm{~dB} /(\mathrm{cm} \sqrt{\mathrm{GHz}})$, and $\alpha_{2}=0.309 \mathrm{~dB} /(\mathrm{cm} \sqrt{\mathrm{GHz}})$, respectively. The input of the structure is connected to a $50-\Omega$ source. The MEF of the device was calculated using (2). The simulated MEF and RF return loss $\left(\left|S_{11}\right|\right)$ using the model in Section II are shown in Fig. 9. The reported theory MEF and $S_{11}$ have been included in Fig. 9 for comparison.

The predicted MEF in Section II for this example is $13.5 \mathrm{~dB}$ at $10 \mathrm{GHz}$. The predicted MEF in [9] is $11.4 \mathrm{~dB}$ at $10 \mathrm{GHz}$. The result in Section II is comparable to the reported MEF, however, the predicted value in [9] was calculated using an approximate closed-form tool. Thus, the numerical model proposed in Section II provides a flexible and realistic approach to implement an RE-MZM device.

The $S_{11}$ value using the model in Section II and in [9] are -19 and $-20 \mathrm{~dB}$, respectively, at $10 \mathrm{GHz}$. The RF return loss in Section II and in the literature have good agreement. Thus, the predicted result in Fig. 9 provides a better accuracy for MEF and $S_{11}$ for the considered device.

\section{E. Summary}

The MEF and $S_{11}$ calculated using the numerical model in Section II were compared with the measured and calculated results reported in the literature. These are summarized in Table I. It is, thus, evident from the results presented in the above sections that improved accuracy can be achieved by using an RF network model in these RE-MZM simulations. Therefore, the proposed model in Section II provides a flexible and realistic design tool for the implementation of RE-MZM devices.

\section{SyNTHESIS OF AN OPTIMIZED RE-MZM DEVICE}

In this section, we use the model in Section II to synthesize an optimized RE-MZM device [5], [20]. This RE-MZM has been fabricated and characterized, and the numerical model is validated through comparison with measurements.

\section{A. Optimized Device Structure}

The RE-MZM configuration is shown in Fig. 10(a). The RE-MZM uses an unenhanced modulator, however, the input (a)

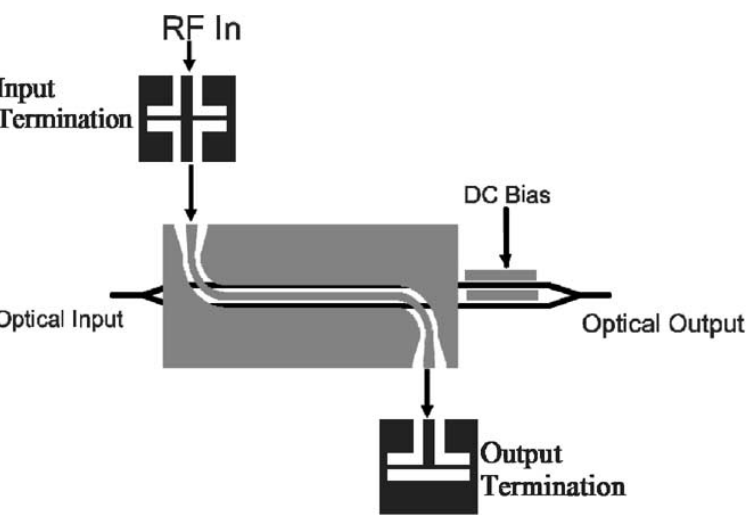

(b)

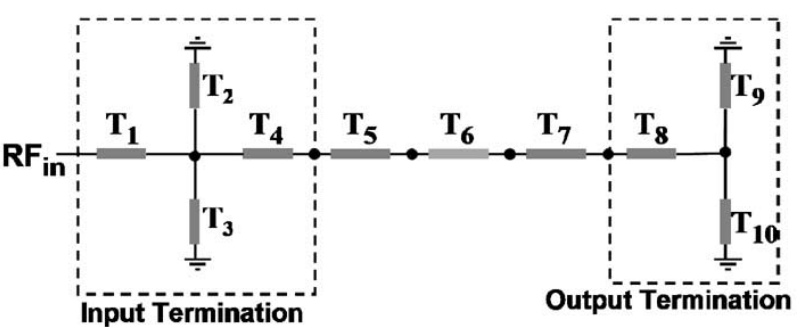

Fig. 10. Schematic of a proposed RE-MZM. (a) Device structure. (b) Equivalent transmission line model.

and output ports are terminated with discontinuities as proposed in [5] and [20]. These discontinuities are the shorted-stub CPW structures as shown in Fig. 10(a). At the input and output ports, two-port and one-port shorted-stub structures are used. These structures were optimized using the numerical model for the corresponding traveling-wave electrode of the modulator as reported in [20] at an operational frequency of $1.8 \mathrm{GHz}$. The numerical tool in Section II provided the various transmission line characteristics of the optimized RE-MZM device.

The equivalent transmission line circuit model of the proposed RE-MZM is shown in Fig. 10(b). In the equivalent model, $T_{6}$ is the active section of the modulator RF electrode, whereas $T_{5}$ and $T_{7}$ are nonactive taper and bend sections of the RF modulator electrode. The shorted stubs are represented by $T_{2}$, $T_{3}, T_{9}$, and $T_{10}$. However, the ports of the terminations are represented by $T_{1}, T_{4}$, and $T_{8}$.

\section{B. Fabrication of the RE-MZM}

Having obtained the optimal configuration as described in [20], it was possible to fabricate and characterize each of the transmission line components identified in Fig. 10(b).

The optical modulator and discontinuities were fabricated on X-cut $\mathrm{LiNbO}_{3}$ and Coorstek AD96-R ceramic substrate, respectively. Diffused 7- $\mu \mathrm{m}$ width optical waveguides on $\mathrm{LiNbO}_{3}$ 
TABLE II

Summary of the RF Electrode and Discontinuities for the Proposed RE-MZM as Depicted in Fig. 10(b)

\begin{tabular}{cccccc}
\hline Device Structure & $\begin{array}{c}L \\
(\mathrm{~mm})\end{array}$ & $\begin{array}{c}Z_{o} \\
(\Omega)\end{array}$ & $N_{m}$ & $\begin{array}{c}\alpha_{o} \\
(\mathrm{~dB} /(\mathrm{cm} \cdot \sqrt{\mathrm{GHz}}))\end{array}$ & $\begin{array}{c}\alpha_{d} \\
(\mathrm{~dB} /(\mathrm{cm} \cdot G H z))\end{array}$ \\
\hline \hline $\mathrm{T}_{6}$ & 37 & 36.24 & 3.23108 & 0.215 & 0.013 \\
$\mathrm{~T}_{5}, \mathrm{~T}_{7}-$ Tapers & 0.6 & 36.24 & 7.48 & 0.215 & 0.013 \\
$\mathrm{~T}_{5}, \mathrm{~T}_{7}-$ Bends & 0.471 & 36.24 & 3.23108 & 0.215 & 0.013 \\
$\mathrm{~T}_{1}, \mathrm{~T}_{4}, \mathrm{~T}_{8}$ & 4.0 & 50.01 & 2.22565 & 0.0371 & 0.0081 \\
$\mathrm{~T}_{2}, \mathrm{~T}_{3}, \mathrm{~T}_{9}, \mathrm{~T}_{10}$ & 3.392 & 143.5 & 2.16739 & 0.0913 & 0.008 \\
\hline
\end{tabular}

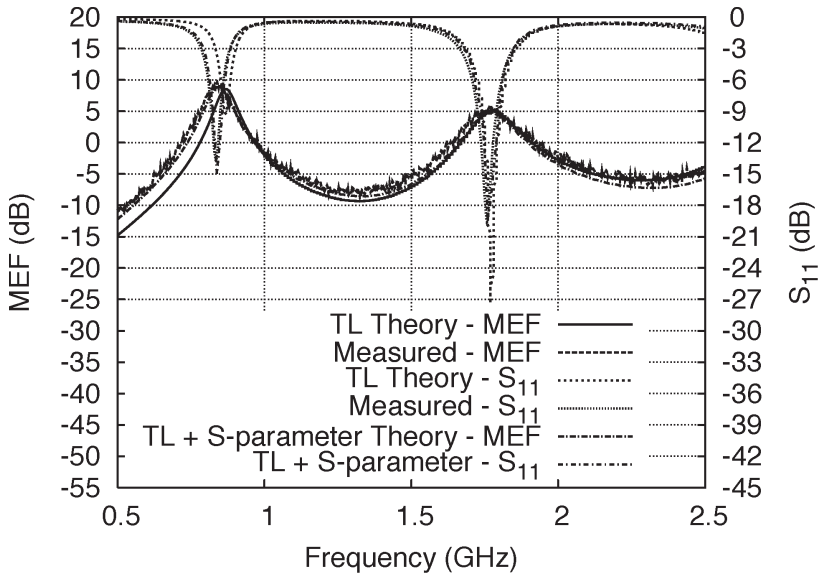

Fig. 11. Comparison of simulated and measured MEF and $S_{11}$ of the structures shown in Figs. 10 and 12, respectively.

was fabricated for single-mode operation at $1550 \mathrm{~nm}$. The transmission line characteristics of the modulator and discontinuities were characterized using RF measurements. The various transmission line sections of the modulator and discontinuities were fabricated and tested for the transmission line characteristics namely, $Z_{o}, N_{m}, \alpha_{o}$, and $\alpha_{d}$.

A summary of the parameters of the shorted-stub terminations and RF electrode are given in Table II, where $\alpha_{d}$ is the dielectric loss of the material. Wire bonding was utilized for the assembly of the optical modulator and the discontinuities.

\section{Experimental Characterization}

The RF performance of the assembled device was measured using an $\mathrm{RF} /$ photonic probe station. The modulator was biased at quadrature. The simulated MEF and RF return loss are shown in Fig. 11 as presented in [20]. Link gain and RF return loss for the optical modulator with and without discontinuities were measured. The measured MEF was normalized using (2). The measured MEF and RF return loss are also shown in Fig. 11. The measured and simulated MEF and $S_{11}$ are in good agreement, however, a small frequency shift exists. This was anticipated due to the wire bond during the assembly, which was not accounted for in the numerical model.

A more detailed representation of the assembled device is shown in Fig. 12. This is the equivalent transmission line model based on a two-port S-parameter at the input and one-port $\mathrm{S}$-parameter at the output. The S-parameter was measured for each of the discontinuities and with the equivalent transmission line model for the modulator, a more realistic simulation was performed. In the model, interconnect point $\left(I_{\text {in }}, I_{\text {out }}\right)$ were

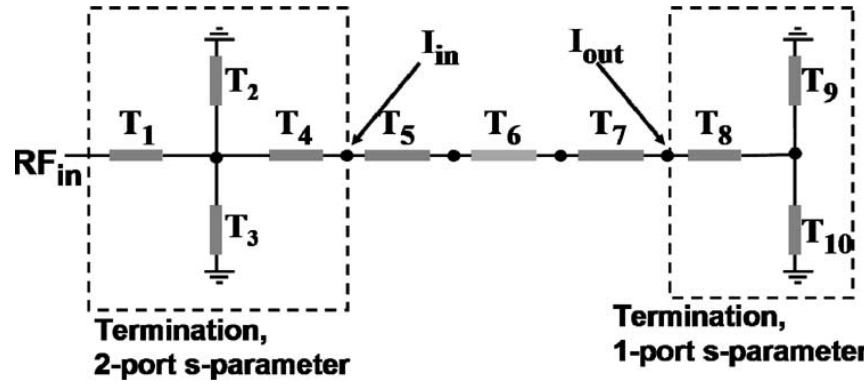

Fig. 12. Equivalent transmission line and S-parameter data model for configuration shown in Fig. 10(a).

placed with $0.25-\mathrm{nH}$ inductive elements to represent the wire bond between the discontinuities and the RF electrode.

The numerical solution for the improved model using Section II is shown in Fig. 11. The measured and simulated results have good agreement in performance as well as the operational frequency. The proposed RE-MZM has an improvement of approximately $5 \mathrm{~dB}$ at $1.8 \mathrm{GHz}$, however, at about $850 \mathrm{MHz}$, the improvement is $\sim 10 \mathrm{~dB}$. The predicted and measured $\mathrm{RF}$ return losses are better than $-10 \mathrm{~dB}$, respectively. However, $S_{11}$ is better at $1.8 \mathrm{GHz}$ than at $850 \mathrm{MHz}$, since the RE-MZM was optimized at $1.8 \mathrm{GHz}$.

\section{Summary}

In this section, numerical design approach was applied to the synthesis of an optimized RE-MZM. The optical modulator and the discontinuities were fabricated based on the required transmission line characteristics. Thereafter, the assembled device was measured and compared with the predicted numerical model performance.

The performance predicted using our design approach is in excellent agreement with the measured results. Further, the successful incorporation of this design sequence into an automatic numerical optimization routine demonstrates its accuracy, flexibility, and robustness.

This demonstration represents a fairly simple resonantly enhanced modulator structure. The approach, however, is capable of simulating arbitrarily complex networks of electrodes and optical waveguides. Thus, the encouraging results achieved in this investigation should lead to the synthesis and analysis of more sophisticated modulator structures in future investigations.

\section{Conclusion}

A systematic design approach of arbitrary configuration of RE-MZM can be achieved using the developed numerical 
model. This model extends the application of available electronic network simulators in order to analyze electrooptic devices, since it provided a flexible approach to the design of RE-MZMs. Previously reported RE-MZM structures have been used as numerical examples to validate the model.

An optimized RE-MZM was synthesized using the numerical optimization and the design approach, and, later, the RE-MZM was fabricated. Modulation enhancement factor and RF return loss was measured, and good agreement was achieved with prediction.

The design approach using the developed numerical tool is capable of analyzing arbitrary electrical and optical networks, and, thus, more sophisticated resonant modulator configurations will be considered in future investigations.

\section{ACKNOWLEDGMENT}

The authors thank D. Pavlickovski, P. Jones, M. Miljanic, Y. Cao, C. Wu, and B. Kealy for fabricating the superstrates, optical modulators, and modulator carrier, and for the characterization of the devices using the facilities of the MMTC, School of Electrical and Computer Engineering at RMIT University.

\section{REFERENCES}

[1] E. L. Wooten, K. M. Kissa, A. Yi-Yan, E. J. Murphy, D. A. Lafaw, P. F. Hallemeier, D. Maack, D. V. Attanasio, D. J. Fritz, G. J. McBrien, and D. E. Bossi, "Review of lithium niobate modulators for fiber-optic communications systems," IEEE J. Sel. Topics Quantum Electron., vol. 6, no. 1, pp. 69-82, Jan./Feb. 2000.

[2] G. L. Li and P. K. L. Yu, "Optical intensity modulators for digital and analog applications," J. Lightw. Technol., vol. 21, no. 9, pp. 2010-2030, Sep. 2003.

[3] N. Dagli, "Wide-bandwidth lasers and modulators for RF photonics," IEEE Trans. Microw. Theory Tech., vol. 47, no. 7, pp. 1151-1171, Jul. 1999.

[4] A. Nirmalathas, D. Novak, C. Lim, R. Waterhouse, and D. Castleford, "Fiber networks for wireless applications," in 13th IEEE Annu. Meeting Lasers and Electro-Optics Society (LEOS), Rio Grande, PR, 2000, vol. 1, pp. 35-36.

[5] Y. S. Visagathilagar, A. Mitchell, and R. B. Waterhouse, "Fabry-Pérot type resonantly enhanced Mach-Zehnder modulator," in IEEE Int. Topical Meeting Microwave Photonics, Melbourne, Australia, 1999, pp. 17-20.

[6] G. K. Gopalakrishnan and W. K. Burns, "Performance and modeling of resonantly enhanced $\mathrm{LiNbO}_{3}$ modulators for low-loss analog fiber-optic links," IEEE Trans. Microw. Theory Tech., vol. 42, no. 12, pp. 2650-2656, Dec. 1994.

[7] T. Kawanishi, S. Oikawa, K. Higuma, Y. Matsuo, and M. Izutsu, " $\mathrm{LiNbO}_{3}$ resonant-type optical modulator with double-stub structure," Electron. Lett., vol. 37, no. 20, pp. 1244-1246, Sep. 2001.

[8] R. Krahenbuhl and M. M. Howerton, "Investigations on short-path-length high-speed optical modulators in $\mathrm{LiNbO}_{3}$ with resonant-type electrodes," J. Lightw. Technol., vol. 19, no. 9, pp. 1287-1297, Sep. 2001.

[9] T. Kawanishi, S. Oikawa, K. Higuma, M. Sasaki, and M. Izutsu, "Design of $\mathrm{LiNbO}_{3}$ optical modulator with asymmetric resonant structure," IEICE Trans. Electron., vol. E85-C, no. 1, pp. 150-155, Jan. 2002.

[10] H. F. Taylor, "Enhanced electrooptic modulation efficiency utilizing slow-wave optical propagation," J. Lightw. Technol., vol. 17, no. 10, pp. $1875-1883$, Oct. 1999.

[11] G. K. Gopalakrishnan, W. K. Burns, R. W. McElhanon, C. H. Bulmer, and A. Greenblatt, "Performance and modeling of broadband $\mathrm{LiNbO}_{3}$ traveling wave optical intensity modulators," J. Lightw. Technol., vol. 12, no. 10, pp. 1807-1819, Oct. 1994.

[12] R. Krahenbuhl and W. K. Burns, "Modelling of broad-band travellingwave optical-intensity modulators," IEEE Trans. Microw. Theory Tech., vol. 48, no. 5, pp. 860-864, May 2000.

[13] C. Collado, O. Menendez, M. C. Santos, J. Mateu, and J. M. O'Callaghan, "General equations for the induced phase shift in resonant electrooptic modulators," IEEE Photon. Technol. Lett., vol. 17, no. 2, pp. 330-332, Feb. 2005.

[14] Y. S. Visagathilagar, A. Mitchell, and M. W. Austin, "General theoretical model for resonantly enhanced optical modulators," in Proc. 8th Optoelectronics and Communications Conf. (OECC), Shanghai, China, Oct. 2004, pp. 337-338.

[15] M. Pirola, F. Cappelluti, G. Giarola, and G. Ghione, "Multisectional modeling of high-speed electrooptic modulators integrated in a microwave circuit CAD environment," J. Lightw. Technol., vol. 21, no. 12, pp. 2989-2996, Dec. 2003.

[16] D. M. Pozar, Microwave Engineering, 2nd ed. New York: Wiley, 1998.

[17] R. N. Simons, Coplanar Waveguide Circuits, Components and Systems. New York: Wiley, 2001.

[18] J. Jin, The Finite Element Method in Electromagnetics. New York: Wiley, 1993.

[19] K. Kawano and T. Kitoh, Introduction to Optical Waveguide Analysis: Solving Maxwell's Equation and the Schrödinger Equation. New York: Wiley, 2001.

[20] T. G. Nguyen, A. Mitchell, and Y. S. Visagathilagar, "Investigation of resonantly enhanced modulators on $\mathrm{LiNbO}_{3}$ using FEM and numerical optimization technique," J. Lightw. Technol., vol. 22, no. 2, pp. 526-533, Feb. 2004.

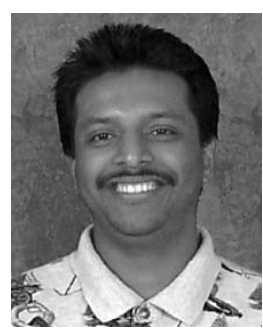

Yuvaraja S. Visagathilagar (S'93-A'02-M'02) was born in Colombo, Sri Lanka, on July 23, 1972. $\mathrm{He}$ received the B.Eng. degree in communications (with first-class honors) and the Ph.D. degree in communication engineering from the Royal Melbourne Institute of Technology (RMIT), Melbourne, Australia, in 1996 and 2003, respectively.

From 1999 to 2002, he was a Researcher undertaking the development of high-speed optical modulators as part of a defense contract. During 2002-2004 he was undertaking research at the Australian Photonics CRC, RMIT University. He is currently a Research Fellow with the School of Electrical and Computer Engineering, RMIT University, where he investigates broadband and specialized optical modulators, in particular, narrowband and resonantly enhanced modulators. His research interests are in theoretical and experimental investigation of microwave and microwave/photonic devices as well as integrating theses device with microwave devices/antennas for telecommunication and defense applications.

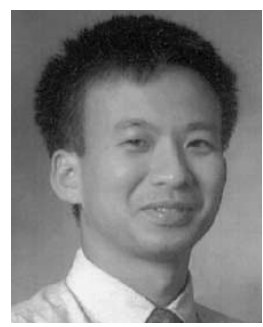

Thach G. Nguyen was born in Quang Tri, Vietnam, in 1975. He received the B.Eng. degree in communication engineering (with first-class honors) from the Royal Melbourne Institute of Technology (RMIT), Melbourne, Australia, in 1998. He is currently working toward the Ph.D. degree in communication engineering at the School of Electrical and Computer Engineering, RMIT

From 1999 to 2002, he was a Design Engineer with VITECO, Vietnam Post and Telecom Corporation. His research interests include the investigation of specialized optical modulators.

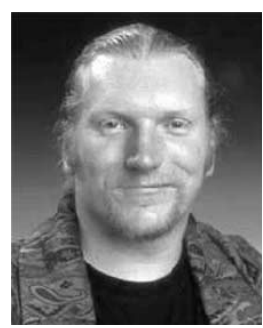

Arnan A. Mitchell (S'97-M'00) was born in Dublin, Ireland, on February 20, 1973. He received the B.Tech. degree in optoelectronics (with honors) from Macquarie University, Sydney, Australia, in 1993 and the Ph.D. degree from the Royal Melbourne Institute of Technology (RMIT), Melbourne, Australia.

He was an Australian Photonics CRC Research Fellow at RMIT investigating broadband and specialized integrated optical modulators and radio frequency (RF) photonic components for communications and signal-processing applications. Currently, he is a Senior Lecturer in the School of Electrical and Computer Engineering, RMIT. He maintains an active interest in the research of numerical methods required for the design of RF photonic integrated devices. 


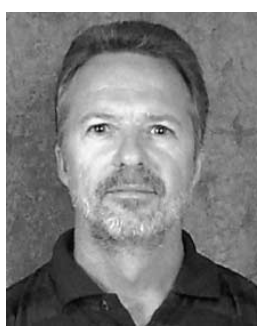

Michael W. Austin (M'83) received the B.Eng. degree in communication engineering (with distinction) and the master's degree in electronic engineering from the Royal Melbourne Institute of Technology (RMIT), Melbourne, Australia, in 1977 and 1980, respectively, and the Ph.D. degree in electronic engineering from the University of London, London, U.K., in 1982.

From 1979 to 1982, he was a Research Fellow with the British Telecom Research Laboratories, Ipswich, U.K., where he was investigating integrated optic devices fabricated in III-V semiconductors. Since 1982, he has been a member of Academic Staff of the Department of Communication and Electronic Engineering, RMIT (now the School of Electrical and Computer Engineering). He has been the Director of RMIT's Microelectronics and Materials Technology Centre (MMTC) since 1994. He is also the Program Manager for the Photonics Information Processing research program in the Australian Photonics Cooperative Research Centre. His research interests include the theoretical and experimental study of planar optical waveguide devices, particularly the fabrication of wideband optical intensity modulators in lithium niobate.

Prof. Austin is a Fellow of the Institution of Engineers, Australia. 